\title{
TREATMENT OF CERTAIN TYPES OF MALOCCLUSION WITH THE AID OF BRIDGE-WORK
}

\author{
By H. E. Bliler, D.D.S., Chicago, Ill.
}

$\mathrm{T}^{\mathrm{H}}$ HE correction of malocclusion must necessarily be considered from different angles and there are certain factors entering into the treatment of one case which may not influence another-the time required to correct the malocclusion, the inflammation created, the inconvenience to patients, their financial status, and the best mode of procedure to obtain quick and lasting results. Every general practitioner should be able to correct simple cases, especially where it is not possible to refer the patient to a specialist, as in the smaller towns and rural districts. It is a well known fact that cases can be treated successfully at a certain age while at a more advanced period of life the treatment of them is much more

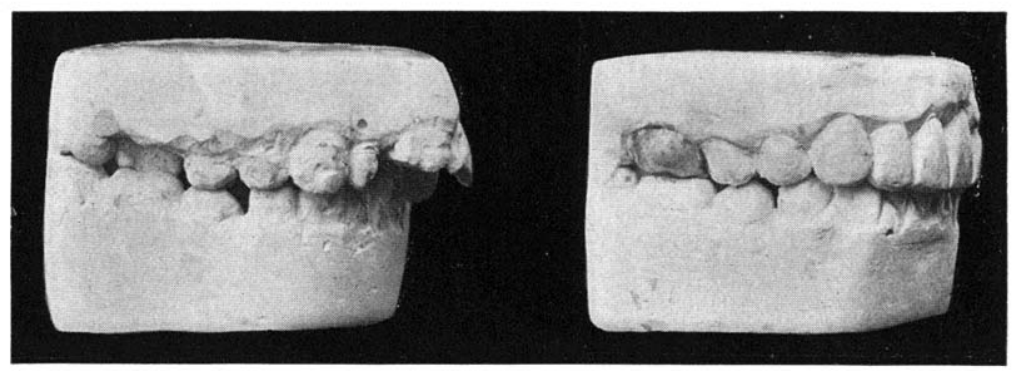

Fig. 1.-Showing models of case before, and after treatment. Fifteen days was allowed for shrinkage or absorption of the V-shaped arch.

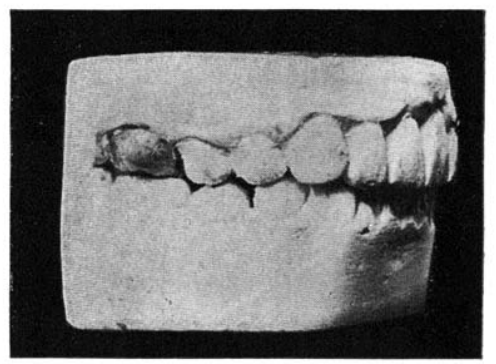

Fig. 2.-Gold crown was placed on six.ycar molar.

troublesome even if not prohibitive. It must also be remembered that certain people can give a considerable length of time to the treatment of malocclusion while others have a limited amount of time and cannot devote several years to the treatment, if there is any other means of accomplishing an improvement in a shorter space of time. It must also be remembered that in some instances the financial inability of the patient to pay for a long treatment, even though the result meant the ideal condition, often causes the operator to decide in favor of a pian whereby he accomplishes results in much less time even though the results are not as ideal as those that require a greater amount of time. 


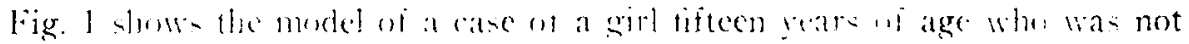
able to spend the necenary lime and money wh he be ase rectifed by ortho-

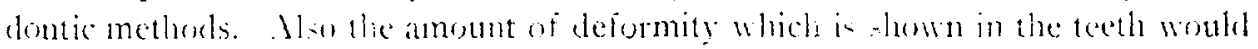

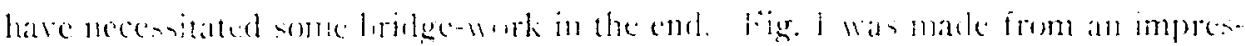

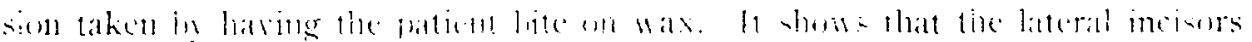

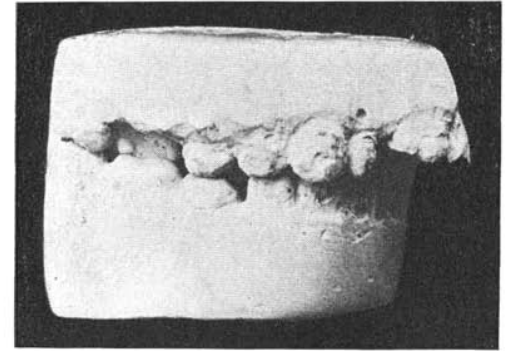

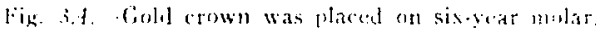

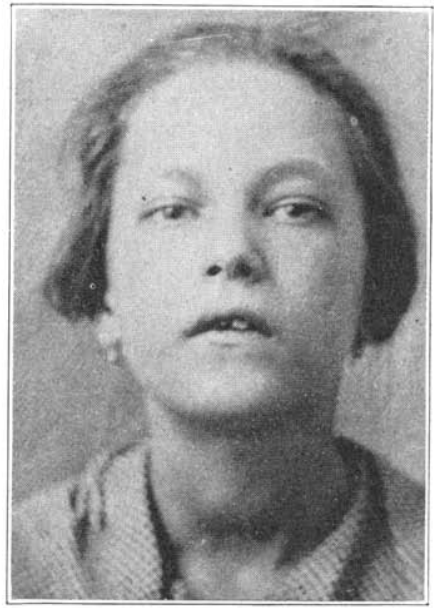

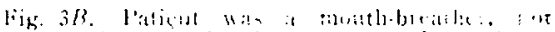

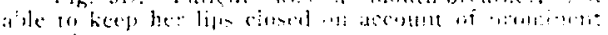
iniralis.

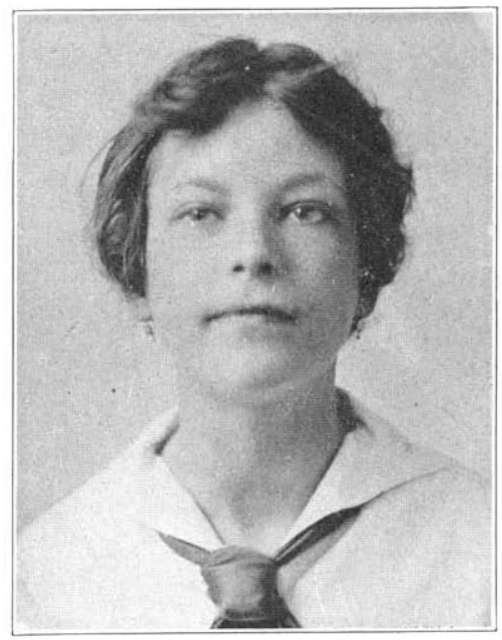

liks. !

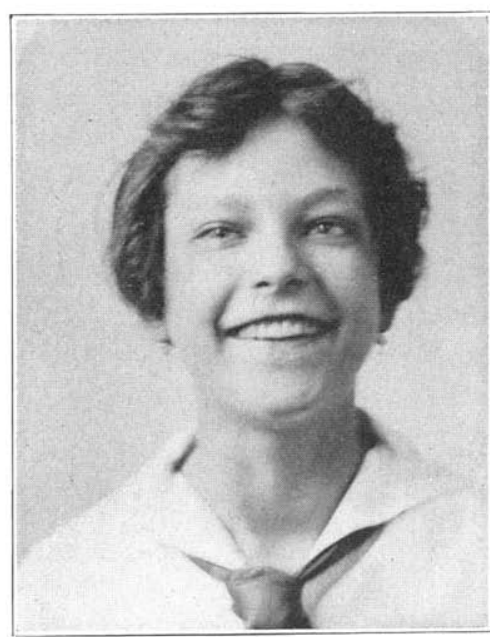

Yis. :

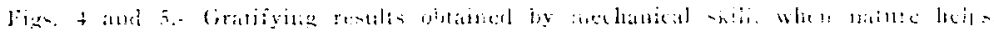

were small, consequently something wat required in order (1) improve the lateral incisors as well as to correct the protrusion of the centrals. Botle centrals. which were oversized, were extracted and the pulp was killel in the two undersized lateral reeth. Feloruary 2)th 1 cut off the lateral teeth and made a funteresth

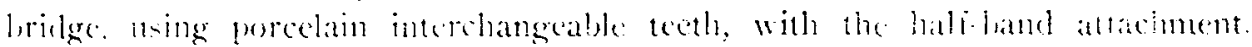


finishing March 3rd, giving the appearance as shown in Fig. 2. One of the central incisors was broken off, the lateral incisors were undersized, and it necessarily made a very unpleasing type of malocclusion, which is shown in Fig. 3. Fig. 4 shows the appearance of the patient's face and lips in repose after the case was treated, while Irig. 5 shows the appearance of the patient's face while smiling. In my opinion it was the correct solution of the casc regardless of her ability to pay more or less.

IVc are aware of the fact that the extraction of an anterior tooth in the correction of malocclusion is to be avoided, but here was a case where the centrals were deformed by being oversized, one of them was broken off, the lateral incisors were small, even if the strictly orthodox plan of orthodontic treatment was followed bridge-work and restoration would have been necessary. This meant time and money as a decided factor, consequently, the method of extracting the two large incisors, and adjusting a four-tooth bridge by using the small lateral incisors as abutment, was decided upon. In fact I am very much in doubt if a letter appearance could have been accomplished considering the patient we had to work on, and the condition in the beginning, if a different plan had been followe 1 However, in the use and the construction of the bridges for the correction of malocclusion, they must be constructed according to anatomical laws, the force of the occlusion must be observed, the operator must be positive that the constructed bridge will be better in appearance than the deformed and mutilated teeth which are extracted.

I get good results many times by extracting the lower central in baclly crowded conditions rather than torturing the patient by trying to widen the arch. or by extracting the first bicuspid and trying to move several teeth over. Py cxtracting the central, the teeth will straighten themselves out, and it is good practice for poor patients, who otherwise would have crowded teeth all their lives. I extracted a lower lateral tocth for a man, 37 years old, and the teeth straightened, without any appliances, in a ycar's time.

\title{
BANDS OR CROWNS FOR TEMPORARY MOLARS
}

\author{
By W. A. Coston, D.D.S., TOPFik, Kansas.
}

$\mathrm{T}^{\mathrm{H}}$ HE difficulties-discomfort and sometimes pain attending the proper fitting and adjusting of anchor bands on molars, especially the temporary molars, is sometimes quite annoying to both the little patient and the dentist, and the fact that they sometimes do not stay on-that they loosen, come off, or are driven into the soft tissuc by the force of occlusion, and cause inflammation of the gingiva, if not the peridental membranc, or sometimes cause etching of the enamel, is also quite annoying. All of these things happen to the "busy dentist," and the following method, which has proved very satisiactory to the author, is suggested for the relief and the comfort of the little patient.

The indirect method of making various restorations has been a great benefaction to dentists--especially the general practitioner, and a great factor in all 